\title{
Los cromosomas meióticos de la rana arborícola Smilisca baudinii (Anura: Hylidae)
}

\author{
Javier Hernández-Guzmán ${ }^{1}$, Lenin Arias-Rodriguez ${ }^{1 *}$ \& Jeane Rimber Indy ${ }^{1,2}$ \\ 1. División Académica de Ciencias Biológicas, Universidad Juárez Autónoma de Tabasco, México; \\ jhernandez-guzman@hotmail.com, leninariasrodriguez@hotmail.com \\ 2. Laboratory of Aquaculture, Campus Bahu, Sam Ratulangi University, Manado, Indonesia; \\ jeanerimberindy@yahoo.com \\ * Correspoondencia
}

Recibido 15-VII-2010. Corregido 10-IX-2010. Aceptado 12-X-2010.

\begin{abstract}
Meiotic chromosomes of the tree frog Smilisca baudinii (Anura: Hylidae). The Mexican tree frog Smilisca baudinii, is a very common frog in Central America. In spite their importance to keep the ecological equilibrium of the rainforest, its biology and genetics are poorly known. In order to contribute with its biological knowledge, we described the typical meiotic karyotype based in standard cytogenetic protocols to specimens collected in Tabasco, Mexico. The study was centered in the analysis of 131 chromosome spreads at meiotic stage from two adults of the species (one female and one male). The metaphase analysis allowed the establishment of the modal haploid number of $1 n=12$ bivalent chromosomes. The chromosomic formulae from the haploid bivalent karyotype was integrated by 12 biarmed chromosomes characterized by twelve pairs of metacentric-submetacentric ( $\mathrm{msm}$ ) chromosomes. The meiotic counting gives the idea that diploid chromosome number is integrated by a complement of $2 n=24$ biarmed chromosomes. The presence of sex chromosomes from female and male meiotic spreads was not observed. Current results suggest that $S$. baudinii chromosome structure is well shared among Hylidae family and "B" chromosomes are particular structures that have very important evolutionary consequences in species diversification. Rev. Biol. Trop. 59 (1): 355-362. Epub 2011 March 01.
\end{abstract}

Key words: Hylidae, Smilisca baudinii, karyotype, chromosome, meiosis.

En México, se ha reportado una amplia diversidad de anfibios con 290 especies registradas, ocupando el cuarto lugar en el mundo (Faivovich et al. 2005, Sánchez \& Barba 2005). De dichas especies, diez se han registrado en el sureste de México particularmente en Tabasco (Reynoso-Rosales et al. 2005). Del grupo de los anuros (ranas y sapos) la familia Hylidae es la que posee mayor diversidad, con un total de 870 especies reportadas en el planeta hasta el 2005 (Faivovich et al. 2005, Frost et al. 2006). Los estudios sobre anfibios, se han basado principalmente en descripciones taxonómicas, distribución geográfica y dinámica poblacional (Busin et al. 2001, 2008, Duellman 1956,
Faivovich et al. 2005, Reynoso-Rosales et al. 2005, Santos et al. 2009, Lynch 2006, Wiens et al. 2005). En lo que respecta a estudios de citogenética, son poco frecuentes en el grupo a pesar de ser caracteres importantes en muchos casos, clave para la comprensión de varios aspectos de biología básica, la dilucidación de problemas taxonómicos, procesos evolutivos, hibridación y mecanismos de determinación del sexo (Ananias et al. 2004, Busin et al. 2001, 2008, Córdova 1993, Faivovich et al. 2005, Gruber et al. 2005). Por otro lado, se ha encontrado que los estudios de citogenética, son un instrumento importante para la detección de daño al genoma por probable 
contaminación del medio acuático (AriasRodriguez et al. 2007, 2008, Álvarez-Morales 2009, D’artola-Barceló 2009, Hernández-Guzmán 2009, Herrera-Navarrete 2009).

En México, los estudios de citogenética de anfibios, se restringen al trabajo del sapo común Chaunus marinus (Hernández-Guzmán 2009). Mientras que en el resto de especies, los fundamentos básicos de citogenética continúan como una incógnita, que es importante discernir en estudios posteriores para el establecimiento de planes de conservación y aprovechamiento de las especies nativas, por ello en este documento se presenta por primera ocasión la estructura de los cromosomas meióticos de la rana arborícola mexicana $S$. baudinii.

\section{MATERIALES Y MÉTODOS}

Sitio de recolecta y clasificación taxonómica: Dos especímenes adultos de $S$. baudinii, una hembra y un macho, fueron recolectados en la zona urbana de la Villa Luís Gil Pérez $\left(17^{\circ} 52^{\prime} 43.05^{\prime \prime} \mathrm{N}-93^{\circ} 04^{\prime} 25.03^{\prime \prime} \mathrm{W}\right)$ en el municipio del Centro de Tabasco. La determinación taxonómica de los ejemplares, se realizó con base en los caracteres merísticos y morfométricos señalados por Flores-Villela et al. (1995).

Procedimiento citogenético: La técnica citogenética empleada, fue una adaptación de la recomendada por Arias-Rodriguez et al. (2008, 2009). Luego de dos días posteriores a su captura, los especímenes fueron tratados por seis horas con $40 \mu \mathrm{g} / \mathrm{g}$ de peso de una solución de colchicina al $0.1 \%$ disuelta en citrato de sodio al $0.1 \%$ y posteriormente, fueron sacrificados por hipotermia obteniéndose el tejido gonádico. Los tejidos, fueron hidratados en una solución de citrato de sodio al $1.0 \%$ y durante $60 \mathrm{~min}$ a $38.0 \pm 1.0^{\circ} \mathrm{C}$; prefijados adicionando (en proporción 1:1) al agente hidratante, la solución de metanol frío $\left(4^{\circ} \mathrm{C}\right)$ : ácido acético $(4: 1)$ y mantenidos en refrigeración $\left(4.0^{\circ} \mathrm{C}\right)$ durante $96 \mathrm{hr}$. Posteriormente, los tejidos fueron limpiados por medio del centrifugado a $4.0^{\circ} \mathrm{C}$ durante $10 \mathrm{~min}$ a $7000 \mathrm{rpm}$ y el sobrenadante fue reemplazado por el fijador $4: 1$. La operación anterior, se repitió por cinco veces hasta que los tejidos adoptaron coloración blanquecina y fueron mantenidos en refrigeración $\left(4^{\circ} \mathrm{C}\right)$ por mes y medio.

Los tejidos fijados, se gotearon sobre series de portaobjetos que fueron previamente colocados en etanol refrigerado $\left(4^{\circ} \mathrm{C}\right)$, y desde una altura aproximada de $1.70 \mathrm{~m}$, lo que permitió la dispersión adecuada de los cromosomas.

Las preparaciones, fueron teñidas con giemsa diluida al $10 \%$ en buffer de fosfatos a pH 7.0 durante $30 \mathrm{~min}\left(38.0 \pm 1.0^{\circ} \mathrm{C}\right)$ y analizadas con el microscopio Zeizz Axiostar Plus con el objetivo 40X. Las mejores dispersiones cromosómicas, fueron fotodigitalizadas con la cámara DSC-W30-Sony con el objetivo 100X. Todas las imágenes, fueron mejoradas con el empleo de los filtros RBG del programa Adobe Photoshop CS $8.01^{\odot}$.

Análisis de datos y construcción del cariotipo: El número modal (M) haploide de cromosomas, fue establecido con base en la frecuencia de conteos cromosómicos sobre las imágenes digitalizadas y empleando el programa Adobe Photoshop CS $8.01^{\circ}$. Las dispersiones cromosómicas menos encimadas y bien dispersas, se imprimieron en papel de alta resolución y los cromosomas fueron recortados individualmente para medir la longitud en micrómetros $(\mu \mathrm{m})$ del brazo corto (p) y largo (q). A cada par cromosómico, les fue calculada la media, desviación estándar y longitud relativa (LR=longitud en $\mu \mathrm{m}$ del par cromosómico/longitud total de complemento cromosómico en $\mu \mathrm{m}$ [100]).

El cariotipo, se construyó considerando la proporción de brazos $\mathrm{r}=\mathrm{q} / \mathrm{p}$, índice centromérico $\mathrm{i}=100(\mathrm{p} / \mathrm{p}+\mathrm{q})$ y la diferencia entre brazos $\mathrm{d}=\mathrm{r}-1(10 / \mathrm{r}+1)$ (Levan et al. 1964). El ideograma en meiosis, se trazó con el programa Microsoft ${ }^{\oplus}$ Office Excel 2003 y basado en las longitudes promedio en $\mu \mathrm{m}$ de $\mathrm{p}+\mathrm{q}$ de cada par cromosómico.

\section{RESULTADOS}

Veintidós preparaciones cromosómicas, del tejido gonádico se observaron 
microscópicamente y fueron fotodigitalizadas 131 dispersiones cromosómicas en meiosis. Del análisis anterior, 96 células mostraron el número haploide de $1 \mathrm{n}=12$ cromosomas bivalentes, lo que representó el $73.3 \%$ (y la moda haploide) del total de dispersiones analizadas (Fig. 1).

Por otro lado, se observaron en ambos especímenes y con baja frecuencia variaciones en los conteos del número de cromosomas de 13, 14 y 24 elementos cromosómicos (Fig. 1, 2).

A partir de las observaciones microscópicas, se logró observar dos estándares de dispersiones cromosómicas en meiosis II. La primera, se caracterizó por mostrar cromosomas bivalentes en forma de anillo en profase temprana, típico de la ovogénesis (Fig. 3A) y la segunda por la presencia de cromosomas bivalentes en metafase II, que caracteriza la espermatogénesis (Fig. 3B).

Las longitudes promedio en micrométros de cada uno de los doce pares cromosómicos del cariotipo profasico, se aprecian en el Cuadro 1. Las medidas variaron desde el par uno con $13.9 \pm 3.45 \mu \mathrm{m}$ y hasta el par doce con $5.10 \pm 0.90 \mu \mathrm{m}$. Mientras, las longitudes promedio de $\mathrm{p}+\mathrm{q}$ de cada uno de los elementos cromosómicos del cariotipo metafásico II, se observan en el Cuadro 2 y al igual que el cariotipo anterior, variaron desde el par uno de $9.60 \pm 2.52 \mu \mathrm{m}$ y hasta el par doce de $4.50 \pm 0.50 \mu \mathrm{m}$.

Basado en las longitudes del complemento cromosómico haploide, se estableció que el cariotipo metafásico de la rana arborícola, se integra por doce cromosomas bivalentes de tipo metacéntrico-submetacéntrico $(\mathrm{msm})$ (Fig. 3B).

En las dispersiones cromosómicas en meiosis del macho y la hembra de $S$. baudinii, no fue posible observar presencia de heteromorfismo cromosómico como criterio para establecer la probable presencia de cromosomas sexuales en la especie.

\section{DISCUSIÓN}

Pese a la inexistencia de estudios de citogenética en las ranas de México, los resultados del número modal haploide $1 \mathrm{n}=12$ cromosomas en meiosis obtenidos en $S$. baudinii demuestran

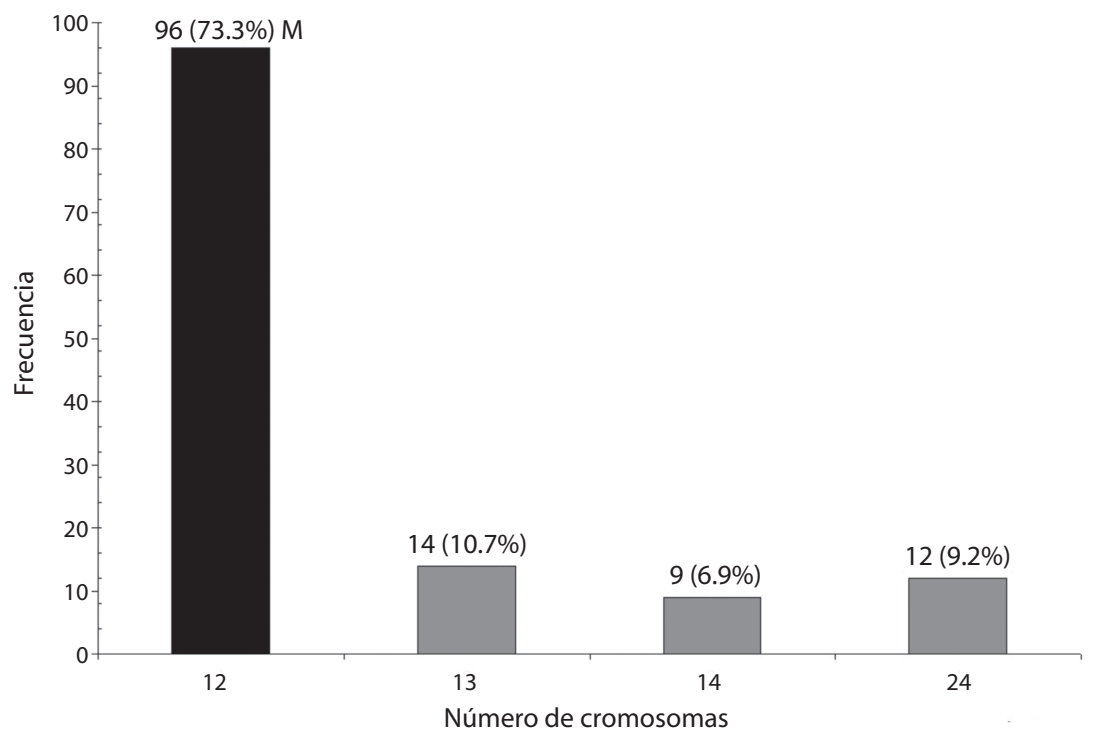

Fig. 1. Frecuencia del número de cromosomas y moda (M) haploide con $1 \mathrm{n}=12$ cromosomas en S. baudinii. Fig. 1. Chromosome number frequency and haploid mode (M) with $1 \mathrm{n}=12$ chromosomes of $S$. baudinii. 

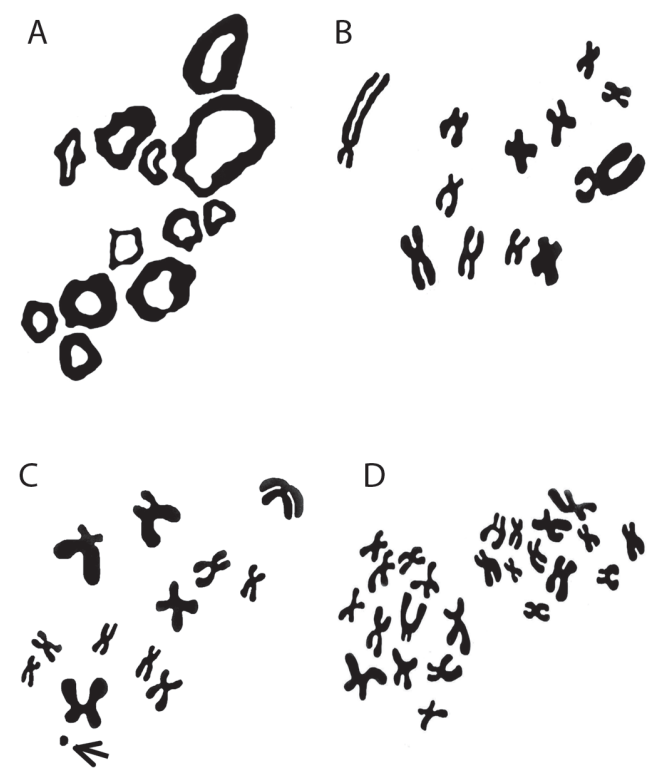

Fig. 2. Dispersiones cromosómicas bivalentes tempranas de $S$. baudinii con 12 cromosomas profasicos II en forma de anillo (A), 12 cromosomas metafasicos II (B), $12+1$ cromosoma "B" (flecha) en metafase II (C) y 24 cromosomas en metafase I (D).

Fig. 2. Early bivalent chromosome spreads from S. baudinii with 12 chromosomes ring shaped at prophase II (A), 12 chromosomes at methaphase II (B), 12+1 "B" chromosome (arrow) at methaphase II (C) and 24 chromosomes at methaphase I (D).
A
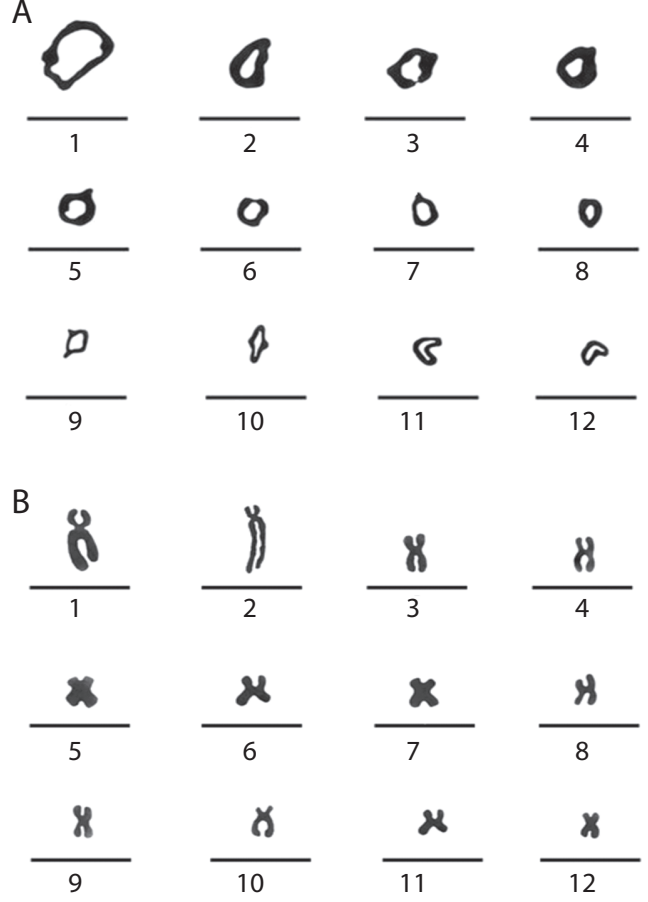

Fig. 3. Cariotipo típico con $1 \mathrm{n}=12$ cromosomas bivalentes en forma de anillo durante la profase temprana en meiosis II de la hembra (A) y cariotipo meiótico del macho de $S$. baudinii en metafase temprana II con $1 \mathrm{n}=12$ cromosomas bivalentes (B).

Fig. 3. Typical karyotype with $1 \mathrm{n}=12$ ring shape bivalent chromosomes at early prophase in meiosis II (A) and meiotic karyotype at early metaphase II stage of $S$. baudinii male with $1 \mathrm{n}=12$ bivalents chromosomes (B).

CUADRO 1

Medidas promedio de los cromosomas bivalentes del cariotipo haploide en profase II de un macho de $\mathrm{S}$. baudini

TABLE 1

Averaged length of the bivalent chromosomes from the haploid karyotype at prophase II of S. baudinii male

$\begin{array}{lcccccccccccc}\text { Par cromosómico } & 1 & 2 & 3 & 4 & 5 & 6 & 7 & 8 & 9 & 10 & 11 & 12 \\ \text { Longitud promedio } & 13.9 \pm & 10.3 \pm & 9.75 \pm & 8.70 \pm & 8.40 \pm & 7.65 \pm & 6.60 \pm & 6.45 \pm & 6.00 \pm & 5.40 \pm & 5.10 \pm & 5.10 \pm \\ \text { en } \mu \text { m } \pm \text { D.E. } & 3.45 & 1.05 & 0.45 & 0.30 & 0.00 & 0.45 & 0.60 & 0.75 & 0.60 & 0.60 & 0.90 & 0.90 \\ & 14.9 \pm & 11.0 \pm & 10.4 \pm & 9.31 \pm & 8.99 \pm & 8.19 \pm & 7.06 \pm & 6.90 \pm & 6.42 \pm & 5.78 \pm & 5.46 \pm & 5.46 \pm \\ \text { L.R. promedio } & 3.18 & 0.06 & 0.77 & 0.84 & 1.26 & 1.83 & 0.07 & 0.93 & 0.02 & 0.11 & 0.61 & 0.61\end{array}$

$\mu \mathrm{m}=$ micrómetros, D.E=desviación estándar, L.R=longitud relativa. 
CUADRO 2

Parámetros citogenéticos promedio del cariotipo típico en meiosis II de la hembra de S. baudinii

TABLE 2

Averaged cytogenetic parameters of the typical karyotype at meiotic stage II from $\mathrm{S}$. baudinii female

$\begin{array}{lccccccc}\begin{array}{c}\text { Par } \\ \text { cromosómico }\end{array} & \begin{array}{c}\text { Longitud promedio en } \\ \mu \mathrm{m} \mathrm{de} \mathrm{p}+\mathrm{q} \pm \mathrm{D} \text {.E. }\end{array} & \begin{array}{c}\mathrm{L} . \mathrm{R} . \\ \mathrm{de} \mathrm{p}\end{array} & \begin{array}{c}\mathrm{L} . \mathrm{R} . \\ \mathrm{de} \mathrm{q}\end{array} & \mathrm{r}=\mathrm{q} / \mathrm{p} & \begin{array}{c}\mathrm{i}= \\ 100(\mathrm{p}) / \mathrm{p}+\mathrm{q}\end{array} & \begin{array}{c}\mathrm{d}= \\ \mathrm{r}-1 * 10 / \mathrm{r}+1\end{array} & \text { Clasificación } \\ 1 & 9.60 \pm 2.25 & 5.08 & 7.93 & 1.56 & 39.0 & 2.19 & \mathrm{msm} \\ 2 & 8.55 \pm 1.49 & 4.88 & 6.71 & 1.38 & 42.1 & 1.58 & \mathrm{msm} \\ 3 & 6.90 \pm 1.24 & 4.47 & 4.88 & 1.09 & 47.8 & 0.43 & \mathrm{msm} \\ 4 & 6.90 \pm 1.14 & 4.27 & 5.08 & 1.19 & 45.6 & 0.87 & \mathrm{msm} \\ 5 & 6.30 \pm 0.50 & 3.86 & 4.67 & 1.21 & 45.2 & 0.95 & \mathrm{msm} \\ 6 & 5.55 \pm 0.50 & 3.46 & 4.67 & 1.35 & 42.5 & 1.50 & \mathrm{msm} \\ 7 & 5.55 \pm 0.67 & 3.46 & 4.07 & 1.18 & 45.9 & 0.81 & \mathrm{msm} \\ 8 & 5.40 \pm 0.84 & 3.25 & 4.07 & 1.25 & 44.4 & 1.11 & \mathrm{msm} \\ 9 & 5.10 \pm 0.72 & 2.85 & 4.07 & 1.43 & 41.1 & 1.76 & \mathrm{msm} \\ 10 & 4.80 \pm 0.60 & 2.85 & 3.66 & 1.29 & 43.7 & 1.25 & \mathrm{msm} \\ 12 & 4.50 \pm 0.50 & 2.64 & 3.46 & 1.31 & 43.3 & 1.33 & \mathrm{msm}\end{array}$

$\mathrm{p}=$ brazo corto, $\mathrm{q}=$ brazo largo, D.E=desviación estándar, L.R=longitud relativa, r=proporción de brazos, i=índice centromérico, $\mathrm{d}=$ diferencia entre brazos, $\mathrm{msm}=$ metacéntrico-submetacéntrico.

que la condición de ploidía reportada, es un parámetro citogenético común en algunos géneros (Hyla, Scinax, Sparasphenodon, Itaponithyla, Hypsiboas) de la familia Hylidae (Cuadro 3) (Faivovich et al. 2005) y probablemente es una condición única para los miembros del género Smilisca.

Faivovich et al. (2005) señala, que los datos citogenéticos disponibles para miembros de la familia Hylidae, se integran por el clado que agrupa a Scarthyla, Scinax, Sphaenorhynchus, "pseudidos", y las Hyla, especies con 30 cromosomas en condición mitótica. Sin embargo, en dicho trabajo, no fueron considerados para el análisis filogenético, los géneros con cariotipo de $2 n=24$ cromosomas y su inclusión, probablemente permitiría reagrupar los clados y relaciones filogenéticas desde la perspectiva citogenética en grupos más acordes con el origen y dispersión de los Hylidae.

Las variaciones del número de elementos cromosómicos señalados en la Fig. 1, son un atributo que ha sido observado únicamente en Hyla sp. (aff. circumdata) (BaldisseraJunior et al. 1993). La presencia de uno o dos cromosomas adicionales al complemento cromosómico haploide en meiosis, se ha interpretado como cromosomas supernumerarios ó cromosomas tipo "B" (Jones \& Rees 1982). Los cromosomas supernumerarios, observados en $S$. baudinii, son los elementos más pequeños $(1.05 \pm 0.15)$ del cariotipo típico de la rana arborícola. Por otro lado, la morfología fue diferente a la del resto de elementos lo que hace suponer su condición univalente en las células meióticas como se ha observado en otras especies (Jones \& Rees 1982, Beukeboom 1994, Palestis et al. 2004). Dichos atributos, ratifican que se trata de cromosomas tipo "B", lo que implica, que su presencia sea de carácter natural y normal en algunas especies de la familia Hylidae.

Los cromosomas "B" supernumerarios, tienen implicaciones importantes durante la historia evolutiva y formación de nuevas especies, dado que la ganancia o perdida de fragmentos de ADN incluyen cambios importantes en la reestructuración del contenido y funcionamiento del genoma de las especies (White 1954, Jones \& Rees 1982, Beukeboom 1994, 
CUADRO 3

Caracterización cariotípica en varias especies de la familia Hylidae

TABLE 3

Karyotype characterization in several species from the Hylidae family

\begin{tabular}{|c|c|c|c|}
\hline Especie & $2 n / 1 n$ & Fórmula & Referencia \\
\hline Smilisca baudinii & $2 \mathrm{n}=? / 1 \mathrm{n}=12$ & $12 \mathrm{msm}$ & Trabajo actual \\
\hline Aparasphenodon brunoi & $2 n=24 / ?$ & $8 m+14 s m+2 s t$ & Nunes \& Fagundes (2008) \\
\hline Itapotihyla langsdorffii & $2 n=24 / ?$ & $12 m+10 s m+2 s t$ & Nunes \& Fagundes (2008) \\
\hline Hypsiboas polytaenius & $2 n=24 / ?$ & $12 \mathrm{~m}+10 \mathrm{sm}+2 \mathrm{st}$ & Nunes \& Fagundes (2008) \\
\hline Scinax alter & $2 n=24 / ?$ & $12 \mathrm{~m}+10 \mathrm{sm}+2 \mathrm{st}$ & Nunes \& Fagundes (2008) \\
\hline Scinax argyreornatus & $2 n=24 / ?$ & $10 m+12 s m+2 s t$ & Nunes \& Fagundes (2008) \\
\hline Scinax perereca & $2 n=24 / 1 n=12$ & $12 \mathrm{~m}+12 \mathrm{sm}$ & Pombal et al. (1995) \\
\hline Hyla savignyi & $2 n=24 / ?$ & $8 m+12 s m+4 s t$ & Martirosyan \& Stepanyan (2007) \\
\hline Hyla schelkownikovi & $2 n=24 / ?$ & $12 m+8 s m+4 s t$ & Martirosyan \& Stepanyan (2007) \\
\hline Hyla berthalutzae & $2 n=30 / ?$ & $12 \mathrm{~m}+10 \mathrm{sm}+8 \mathrm{t}$ & Gruber et al. (2005) \\
\hline Hyla cruzi & $2 \mathrm{n}=30 / ?$ & $14 m+10 s m+6 t$ & Gruber et al. (2005) \\
\hline Hyla elegans & $2 n=30 / ?$ & $10 m+12 s m+8 t$ & Gruber et al. (2005) \\
\hline Hyla elianeae & $2 n=30 / ?$ & $16 m+8 s m+6 t$ & Gruber et al. (2005) \\
\hline Hyla microps & $2 n=30 / ?$ & $14 m+8 s m+8 t$ & Gruber et al. (2005) \\
\hline Hyla nahdereri & $2 n=30 / ?$ & $14 m+6 s m+10 t$ & Gruber et al. (2005) \\
\hline Hyla rubicundula & $2 n=30 / ?$ & $16 m+8 s m+6 t$ & Gruber et al. (2005) \\
\hline Hyla marginata & $2 n=24 / ?$ & $10 m+10 s m+2 s t$ & Ananias et al. (2004) \\
\hline Hyla semiguttata & $2 n=24 / ?$ & $10 \mathrm{~m}+10 \mathrm{sm}+2 \mathrm{st}$ & Ananias et al. (2004) \\
\hline Hyla sp. (aff. semiguttata) & $2 n=24 / ?$ & $10 \mathrm{~m}+10 \mathrm{sm}+2 \mathrm{st}$ & Ananias et al. (2004) \\
\hline Hyla nana & $2 n=30 / ?$ & $12 \mathrm{~m}+10 \mathrm{sm}+8 \mathrm{t}$ & Medeiros et al. (2003) \\
\hline Hyla sanborni & $2 n=30 / ?$ & $12 \mathrm{~m}+8 \mathrm{sm}+10 \mathrm{t}$ & Medeiros et al. (2003) \\
\hline Hyla fuscovaria & $2 n=24 / 1 n=12$ & $?$ & Baldissera-Junior et al. (1993) \\
\hline Hyla hayii & $2 n=24 / 1 n=12$ & $?$ & Baldissera-Junior et al. (1993) \\
\hline Hyla prasina & $2 n=24 / 1 n=12$ & $?$ & Baldissera-Junior et al. (1993) \\
\hline Hyla sp. (aff. circumdata) & $2 n=24 / 1 n=12$ & $?$ & Baldissera-Junior et al. (1993) \\
\hline Hyla sp. (aff. circumdata) & $2 n=25 / 1 n=13$ & $?$ & Baldissera-Junior et al. (1993) \\
\hline Hyla arborea japonica & $2 n=24 / 1 n=12$ & $8 m+14 s m+2 s t$ & Seto (1964) \\
\hline
\end{tabular}

$2 \mathrm{n}=$ diploide, $1 \mathrm{n}=$ haploide, ?= no publicado, $\mathrm{msm}=$ metacéntrico-submetacéntrico, $\mathrm{m}=$ metacéntrico, $\mathrm{msm}=$ metacéntricosubmetacéntrico, $\mathrm{sm}=$ submetacéntrico, $\mathrm{st}=$ subtelocéntrico, $\mathrm{t}=$ telocéntrico

Camacho et al. 2000, Fagundes et al. 2004, Granado et al. 2004, Palestis et al. 2004). Lo anterior, probablemente ha sido un mecanismo importante durante la especiación en la familia Hylidae, por ello la ampliación de los estudios de citogenética en otras especies de ranas del sureste de México permitirá mayor comprensión del valor biológico y evolutivo de los cromosomas supernumerarios.
La morfología cromosómica, del cariotipo típico de $S$. baudini en meiosis, se estableció para el complemento haploide como cromosomas birrámeos de tipo metacéntrico-submetacéntrico $(\mathrm{msm})$ que se caracterizaron por presencia de doce pares de cromosomas bivalentes. Los cromosomas birrámeos (m y msm), es una condición común en las especies de la familia Hylidae (Cuadro 3), lo que se traduce 
en relaciones filogenéticas compartidas no solo por mantener número cromosómico similar, si no que además por conservar fórmula cromosómica constante y como se ha manifestado en varias especies de ranas arborícolas de Brasil, Estados Unidos de América, Armenia y Arabia Saudita (Cuadro 3).

En conclusión, el número haploide en la rana arborícola mexicana $S$. baudinii es de $1 \mathrm{n}=12$ cromosomas, por lo que se asume que en condición diploide en mitosis el cariotipo debe estar integrado por 24 cromosomas birrámeos de tipo msm. La presencia de cromosomas sexuales, fue descartada en esta especie, debido a la carencia de atributos morfológicos para identificar heteromorfismo entre las dispersiones meióticas del espécimen hembra y macho.

\section{AGRADECIMIENTOS}

El presente documento tuvo apoyo del rancho las AMAZONAS, ubicado en Villa Luís Gil Pérez, Centro, Tabasco, México otorgado al segundo autor L.A.R.

\section{RESUMEN}

La rana arborícola mexicana Smilisca baudinii, es una especie de rana común en Centroamérica. Sin embargo, la biología y genética de la especie, es pobremente conocida a pesar de su importancia para mantener en equilibrio ecológico las selvas tropicales. Con el propósito de contribuir con el conocimiento biológico de esta especie, establecimos el cariotipo típico en meiosis en especímenes recolectados en Tabasco, México, mediante procedimientos citogenéticos estándares. El estudio, se fundamentó en el análisis de 131 dispersiones cromosómicas en estadio meiótico de dos adultos de la especie (una hembra y un macho). El análisis de las metafases, permitió establecer el número modal haploide de $1 \mathrm{n}=12$ cromosomas bivalentes La fórmula cromosómica del cariotipo haploide, se integró por 12 cromosomas birrámeos caracterizado por 12 pares de cromosomas bivalentes metacéntricos-submetacéntricos (msm). Los conteos en meiosis, hacen suponer como número diploide de cromosomas a un complemento integrado por $2 n=24$ cromosomas birrámeos. No fue posible observar presencia de cromosomas sexuales, entre las dispersiones meióticas del espécimen hembra y macho. Los resultados sugieren que la estructura cromosómica de $S$. baudinii, es compartida ampliamente entre las especies de la familia Hylidae y los cromosomas "B" son estructuras importantes en la diversificación de las especies.
Palabras claves: Hylidae, Smilisca baudinii, cariotipo, cromosoma, meiosis.

\section{REFEERENCIAS}

Álvarez-Morales, M. 2009. Cariotipo de la mojarra paleta Vieja synspila (Hubbs, 1935) en el estado de Tabasco, México. Tesis de Licenciatura, Universidad Juárez Autónoma de Tabasco, Tabasco, México.

Ananias, F., P.C.A. Garcia \& S.M. Recco-Pimentel. 2004. Conserved karyotypes in the Hyla pulchella species group (Anura, Hylidae). Hereditas 140: 42-48.

Arias-Rodriguez, L., J.P. González-Hermoso, H. FletesRegalado, L.E. Rodríguez-Ibarra \& G. Del Valle Pignataro. 2007. Cariotipos de los caracoles de tinte Plicopurpura pansa (Gould, 1853) y Plicopurpura columellaris (Lamarck, 1816) (Gastropoda: Muricidae). Rev. Biol. Trop. 55: 853-866.

Arias-Rodriguez, L., L. Ibarra-Castro \& S. Páramo-Delgadillo. 2008. Los cromosomas mitóticos y meióticos del pez tropical Petenia splendida (Cichlidae). Rev. Biol. Trop. 56: 895-907.

Arias-Rodriguez, L., S. Páramo-Delgadillo, W.M. Contreras-Sánchez \& C.A. Álvarez-González. 2009. Cariotipo del pejelagarto tropical Atractosteus tropicus (Lepisosteiformes: Lepisosteidae) y variación cromosómica en sus larvas y adultos. Rev. Biol. Trop. 57: 529-539.

Baldissera-Junior, F.A., P.S. Lopes de Oliveira \& S. Kasahara. 1993. Cytogenetics of four Brazilian Hyla species (Amphibia-Anura) and description of a case with a supernumerary chromosome. Braz. J. Genet. 16: 335-345.

Beukeboom, L.W.1994. Bewildering Bs: an impression of the 1st B-chromosome conference. Heredity 73 : 328-336.

Busin, C.S., G.Vinciprova \& S.M. Recco-Pimentel. 2001. Chromosomal rearrangements as the source of variation in the number of chromosomes in Pseudis (Amphibia, Anura). Genetica 110: 131-141.

Busin, C.S., G.A. Vasconcellos, J. Bertoldo, M.L. Del Grande, M. Uetanabaro \& S.M. Recco-Pimentel. 2008. Cytogenetic analysis of four species of Pseudis (Anura, Hylidae), with the description of ZZ/ZW sex chromosomes in P. tocantins. Genetica 133: 119-127.

Camacho, J.P.M., T.F. Sharbel \& L.W. Beukeboom. 2000. B-chromosome evolution. Phil. Trans. R. Soc. Lond. B. 355: 163-178. 
Córdova, J.H. 1993. Estudio cariotípicos y problemas taxonómicos en el grupo de Bufo spinulosus (Amphibia: Anura) de Perú. Tesis de Biología, Universidad Nacional Mayor de San Marcos, Lima, Perú.

D'artola-Barceló, A.L. 2009. Aspectos citogenéticos de dos poblaciones de la sardina de agua dulce Astyanax aeneus (Pisces: Characidae). Tesis de Licenciatura, Universidad Juárez Autónoma de Tabasco, Tabasco, México.

Duellman, W.E. 1956. The frogs of the hylid genus Phrynohyas Fitzinger, 1843. Museum of Zoology, University of Michigan 96: 1-68.

Fagundes, V., J.P.M. Camacho \& Y. Yonenaga-Yassuda. 2004. Are the dot-like chromosomes in Trinomys iheringi (Rodentia, Echimyidae) B chromosomes? Cytogenet. Genome. Res. 106: 159-164.

Faivovich, J., C.F.B. Haddad, P.C.A. García, D.R. Frost \& J.A. Campbell. 2005. Systematic review of the frog family Hylidae, with special reference to Hylinae: phylogenetic analysis and taxonomic revision. Bull. Ame. Mus. Nat. Hist. 294: 1-240.

Flores-Villela, O.A., F. Mendoza-Quijano \& G. GonzálezPorter. 1995. Recopilación de claves para la determinación de anfibios y reptiles de México. Publ. Espec. Mus. Zool. Méx.10: 1-285.

Frost, D.R., T. Grant, J. Faivovich, R.H. Bain, A. Haas, C.F.B. Haddad, R.O. De Sá, A. Channing, M. Wilkinson, S.C. Donnellan, C.J. Raxworthy, J.A. Campbell, B.L. Blotto, P. Moler, R.C. Drewes, R.A. Nussbaum, J.D. Lynch, D.M. Green \& W.C. Wheeler. 2006. The Amphibian Tree of Life. Bull. Am. Mus. Nat. Hist. 297: $1-370$

Granado, N., E. Rebollo, F.J. Sánchez \& P. Arana. 2004. B chromosomes: the troubles of integration. Cytogenet. Genome Res. 106: 402-410.

Gruber, S.L., C.F.B. Haddad \& S. Kasahara. 2005. Evaluating the karyotypic diversity in species of Hyla (Anura; Hylidae) with $2 \mathrm{n}=30$ chromosome based on the analysis of ten species. Folia Biologica 51: 68-75.

Hernández-Guzmán, J. 2009. Cariotipo del sapo común Chaunus marinus (Anura: Bufonidae) de Tabasco, México. Tesis de Licenciatura, Universidad Juárez Autónoma de Tabasco, Tabasco, México.

Herrera-Navarrete, M.L. 2009. Estudio citogenético de la mojarra Cichlasoma urophtalmus en dos localidades del estado de Tabasco. Tesis de Licenciatura, Universidad Juárez Autónoma de Tabasco, Tabasco, México.

Jones, R. \& H. Rees. 1982. B chromosomes. Academic, Londres, Inglaterra.
Levan, A., K. Fredga \& A.A. Sandberg. 1964. Nomenclature of centromeric position on chromosomes. Hereditas 52: 201-220.

Lynch, J.D. 2006. The tadpoles of frogs and toads found in the lowlands of northern Colombia. Rev. Acad. Colomb. Cienc. 30: 443-457.

Martirosyan, A. \& I. Stepanyan. 2007. The karyotype of Hyla savignyi Audouin, 1827 (Amphibia: Anura) from Southern Armenia. Comp. Cytogenet. 1: $107-112$

Medeiros, L.R., D.C. Rossa-Feres \& S.M. Recco-Pimentel. 2003. Chromosomal differentiation of Hyla nana and Hyla sanborni (Anura, Hylidae) with a description of NOR polymorphism in H. nana. J. Hered. 94: 149-154.

Nunes, R.R.A. \& V. Fagundes. 2008. Cariótipos de oito espécies de anfíbios das subfamilias Hylinae e Phyllomedusinae (Anura, Hylidae) do Espírito Santo, Brasil. Bol. Mus. Biol. Mello Leitão 23: 21-36.

Palestis, B.G., R. Trivers, A. Burt \& R.N. Jones. 2004. The distribution of B chromosomes across species. Cytogenet. Genome. Res. 106: 151-158.

Pombal, J.P., C.F.B. Haddad \& S. Kasahara. 1995. A new species of Scinax (Anura: Hylidae) from southeastern Brazil, with comments on the genus. J. Herpetol. 29: $1-6$.

Reynoso-Rosales, V.H., F. Mendoza-Quijano, C.S. Valdespino-Torres \& X. Sánchez-Hernández. 2005. Anfibios y reptiles, p. 241-260. In J. Bueno, F. Álvarez \& S. Santiago (eds.). Biodiversidad del estado de Tabasco. Instituto de Biología, UNAM-CONABIO, México.

Sánchez, A.J. \& E. Barba. 2005. Biodiversidad de Tabasco, p. 1-16. In J. Bueno, F. Álvarez \& S. Santiago (eds.). Biodiversidad del estado de Tabasco. Instituto de Biología, UNAM-CONABIO, México.

Santos, J.C., L.A. Coloma, K. Summers, J.P. Caldwell, R. Ree \& D.C. Cannatella. 2009 Amazonian amphibian diversity is primarily derived from late miocene andean lineages. PLoS Biol. 7: 448-461.

Seto, T. 1964. The karyotype of Hyla arborea japonica with some remarks on heteromorphism of the sex chromosome. J. Fac.Sci. Hokkaido U. Serie VI Zoology 15: 366-373.

White, M.J. 1954. Animal Cytogenetics and Evolution. Cambridge, Cambridge, England.

Wiens, J.J., J.W. Fetzner, C.L. Parkinson Jr. \& T.W. Reeder. 2005. Hylid frog phylogeny and sampling strategies for speciose clades. Syst. Biol. 54: 778-807. 